University of Wollongong

Research Online

$1-1-2014$

Comparison of real-time elastography and multiparametric MRI for prostate cancer detection: A whole-mount step-section analysis

Daniel Junker

Medical University Innsbruck

Georg Schafer

Medical University Innsbruck

Conrad Kobel

Medical University Innsbruck, ckobel@uow.edu.au

Christian Kremser

Medical University Innsbruck

Jasmin Bektic

Medical University Innsbruck

See next page for additional authors

Follow this and additional works at: https://ro.uow.edu.au/buspapers

Part of the Business Commons

Research Online is the open access institutional repository for the University of Wollongong. For further information contact the UOW Library: research-pubs@uow.edu.au 


\title{
Comparison of real-time elastography and multiparametric MRI for prostate cancer detection: A whole-mount step-section analysis
}

\begin{abstract}
OBJECTIVE. The purpose of this study was to compare prostate cancer detection rate of real-time elastography (RTE) with that of multiparametric MRI to evaluate the advantages and disadvantages of the two methods. SUBJECTS AND METHODS. Thirty-nine patients with biopsy-proven prostate cancer underwent both RTE and multiparametric MRI to localize prostate cancer before radical prostatectomy. RTE was performed to assess prostate tissue elasticity, and hard lesions were considered suspicious for prostate cancer. Multiparametric MRI included T2-weighted MRI, diffusion-weighted MRI (DWI), and contrast-enhanced MRI (CE-MRI) with an endorectal coil at 1.5 T. After radical prostatectomy, wholemount step sections of the prostate were generated, and the prostate cancer detection rates with both modalities were analyzed for cancer lesions measuring $0.2 \mathrm{~cm} 3$ or larger. RESULTS. Histopathologic examination revealed 61 cancer lesions. RTE depicted 39 of 50 cancer lesions (78.0\%) in the peripheral zone and 2 of 11 (18.2\%) in the transitional zone. Multiparametric MRI depicted 45 of 50 cancer lesions $(90.0 \%)$ in the peripheral zone and 8 of $11(72.7 \%)$ in the transitional zone. Significant differences between the two modalities were found for the transitional zone and anterior part in prostates with volumes greater than $40 \mathrm{~cm} 3(p<0.05)$. Detection rates for high-risk prostate cancer (Gleason score $\geq 4$ and 3 ) and cancer lesions with volumes greater than $0.5 \mathrm{~cm} 3$ were high for both methods $(93.8 \%$ and $80.5 \%$ for RTE, $87.5 \%$ and $92.7 \%$ for multiparametric MRI). Volumetric measurements of prostate cancer were more reliable with T2-weighted MRI than with RTE (Spearman rank correlation, 0.72 and 0.46 ). CONCLUSION. RTE and multiparametric MRI depicted high-risk prostate cancer with high sensitivity. However, multiparametric MRI seems to have advantages in tumor volume assessment and for the detection of prostate cancer in the transitional zone and anterior part within prostates larger than $40 \mathrm{~cm} 3$. American Roentgen Ray Society.
\end{abstract}

\section{Keywords}

detection, section, whole, analysis, cancer, prostate, mri, multiparametric, elastography, time, real, comparison, mount, step

\section{Disciplines \\ Business}

\section{Publication Details}

Junker, D., Schäfer, G., Kobel, C., Kremser, C., Bektic, J., Jaschke, W. \& Aigner, F. (2014). Comparison of real-time elastography and multiparametric MRI for prostate cancer detection: A whole-mount stepsection analysis. American Journal of Roentgenology, 202 (3), W263-W269.

\section{Authors}

Daniel Junker, Georg Schafer, Conrad Kobel, Christian Kremser, Jasmin Bektic, Werner Jaschke, and Friedrich Aigner 


\section{Comparison of Real-Time Elastography and Multiparametric MRI for Prostate Cancer Detection: A Whole-Mount Step-Section Analysis}

\section{Daniel Junker ${ }^{1}$ \\ Georg Schäfer 2,3 \\ Conrad Kobel ${ }^{4}$ \\ Christian Kremser ${ }^{1}$ \\ Jasmin Bektic ${ }^{3}$ \\ Werner Jaschke ${ }^{1}$ \\ Friedrich Aigner ${ }^{1}$}

Keywords: diffusion, elastography, multiparametric MRI, perfusion, prostate cancer

D0I:10.2214/AJR.13.11061

Received April 8, 2013; accepted after revision June 18,2013

'Department of Radiology, Medical University of Innsbruck, Anichstrasse 35, 6020 Innsbruck, Austria. Address correspondence to D. Junker (daniel.junker@uki.at).

${ }^{2}$ Department of Pathology, Medical University of Innsbruck, Innsbruck, Austria.

${ }^{3}$ Department of Urology, Medical University of Innsbruck Innsbruck, Austria.

${ }^{4}$ Department of Medical Statistics, Informatics, and Health Economics, Medical University of Innsbruck, Innsbruck, Austria.

WEB

This is a web exclusive article.

AJR2014; 202:W263-W269

0361-803X/14/2023-W263

(c) American Roentgen Ray Society

OBJECTIVE. The purpose of this study was to compare prostate cancer detection rate of real-time elastography (RTE) with that of multiparametric MRI to evaluate the advantages and disadvantages of the two methods.

SUBJECTS AND METHODS. Thirty-nine patients with biopsy-proven prostate cancer underwent both RTE and multiparametric MRI to localize prostate cancer before radical prostatectomy. RTE was performed to assess prostate tissue elasticity, and hard lesions were considered suspicious for prostate cancer. Multiparametric MRI included T2-weighted MRI, diffusion-weighted MRI (DWI), and contrast-enhanced MRI (CE-MRI) with an endorectal coil at $1.5 \mathrm{~T}$. After radical prostatectomy, whole-mount step sections of the prostate were generated, and the prostate cancer detection rates with both modalities were analyzed for cancer lesions measuring $0.2 \mathrm{~cm}^{3}$ or larger.

RESULTS. Histopathologic examination revealed 61 cancer lesions. RTE depicted 39 of 50 cancer lesions (78.0\%) in the peripheral zone and 2 of $11(18.2 \%)$ in the transitional zone. Multiparametric MRI depicted 45 of 50 cancer lesions $(90.0 \%)$ in the peripheral zone and 8 of $11(72.7 \%)$ in the transitional zone. Significant differences between the two modalities were found for the transitional zone and anterior part in prostates with volumes greater than $40 \mathrm{~cm}^{3}(p<0.05)$. Detection rates for high-risk prostate cancer (Gleason score $\geq 4$ and 3 ) and cancer lesions with volumes greater than $0.5 \mathrm{~cm}^{3}$ were high for both methods $(93.8 \%$ and $80.5 \%$ for RTE, $87.5 \%$ and $92.7 \%$ for multiparametric MRI). Volumetric measurements of prostate cancer were more reliable with T2-weighted MRI than with RTE (Spearman rank correlation, 0.72 and 0.46 ).

CONCLUSION. RTE and multiparametric MRI depicted high-risk prostate cancer with high sensitivity. However, multiparametric MRI seems to have advantages in tumor volume assessment and for the detection of prostate cancer in the transitional zone and anterior part within prostates larger than $40 \mathrm{~cm}^{3}$.

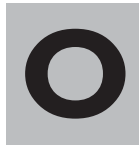
ne of the key requirements of prostate imaging is to support clinicians in managing the diagnosis of and therapy for prostate cancer. The diagnosis of prostate cancer is confirmed by histologic examination of systematic biopsy specimens, but the cancer is not usually visualized with systematic biopsy [1], and therefore prostate cancer may be missed [2]. Most biopsy specimens are obtained from the posterolateral part of the prostate; sampling of the anterior regions and the transitional zone is not recommended, especially in the first systematic biopsy $[1,3]$. Durmus et al. [4] suggest targeted biopsy when multiparametric MRI is performed and novel transrectal ultrasound technologies rather than systematic biopsy.
Besides being useful for tumor localization, prostate imaging provides information about tumor volume, extracapsular extension, seminal vesical invasion, and the degree of cancer aggressiveness. This information may be helpful for choosing the most appropriate therapy, especially focal therapy, active surveillance, and watchful waiting $[5,6]$.

Two imaging modalities have been reported to be reliable for prostate imaging and prostate cancer detection. Real-time elastography (RTE) is an ultrasound technique that provides information about tissue elasticity and color codes hard areas, in blue for example [7]. Prostate cancer has higher cell and vessel density than the normal surrounding tissue and therefore exhibits increased stiffness [8]. RTE can be performed under real-time condi- 


\section{Junker et al.}

tions, which is useful for targeted biopsy [9]. Multiparametric MRI has been introduced to raise the overall diagnostic accuracy in prostate cancer diagnosis by revealing structural and functional tissue information that may help identify prostate cancer [10]. In multiparametric MRI conventional T2-weighted sequences are used to assess structural tissue information, and at least two functional techniques are added, such as diffusion-weighted imaging (DWI) to assess water diffusibility and contrast-enhanced MRI (CE-MRI) to assess contrast dynamics [11].

The aim of this study was to test whetherdepending on tumor localization, clinical significance, or prostate volume-either RTE or multiparametric MRI is superior for the detection of prostate cancer. Although solid data from assessments of each modality separately exist in the literature, in this study we evaluated both modalities in the same cohort of patients. We compared diagnostic performance and estimation of tumor volume for RTE and multiparametric MRI in patients with known prostate cancer using prostate whole-mount step section as the standard of reference. To our knowledge, this is only the second study comparing RTE with multiparametric MRI and the fourth comparing RTE with MRI in general [12-14].

\section{Subjects and Methods Patients}

From April 2010 to January 2012, 40 patients (median age, 62 years; range, 48-75 years) participated in this prospective single-center study. The median serum prostate-specific antigen concentration was $5.2 \mathrm{ng} / \mathrm{mL}$ (range, $2.1-14 \mathrm{ng} / \mathrm{mL}$ ); median prostate volume, $31 \mathrm{~cm}^{3}$ (range, 15-130 $\mathrm{cm}^{3}$ ); and median time between biopsy and imaging, 79 days (range, 40-219 days). A positive vote of the local ethics committee and written informed consent were obtained. All men had biopsy-proven prostate cancer and were scheduled for radical prostatectomy at our institution. After radical prostatectomy the pathologist prepared the prostates as whole-mount step sections and marked the borders of all diagnosed cancer lesions. Thirty-nine of the 40 participants underwent first RTE and then multiparametric MRI the day before radical prostatectomy. The excluded patient underwent RTE but not MRI because of a scheduling conflict. Thirty-eight of the 39 patients underwent all three MRI techniques: T2-weighted MRI, DWI, and CE-MRI. Because of feeling uncomfortable during the examination, one patient underwent only T2-weighted-MRI and DWI but was not excluded from statistical analysis.
Fig. 1-62-year-old man with prostate cancer. Real-time elastogram shows two hard lesions (calipers, blue) in peripheral zone of prostate. D1, D2 = diameters of first lesion. D3 = long diameter of second lesion.

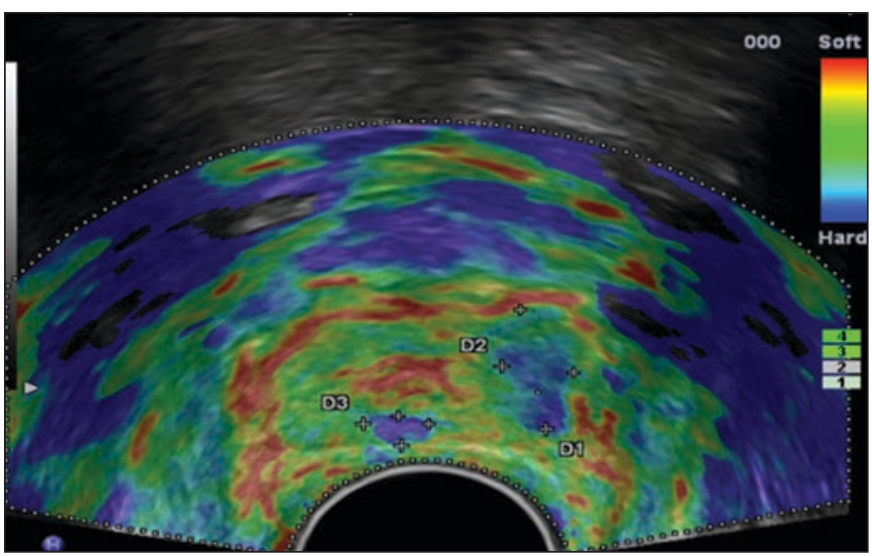

\section{2-Time Elastography}

RTE was performed by an experienced operator using a $7.5-\mathrm{MHz}$ end-firing transrectal probe and an EUB 8500 ultrasound unit (Hitachi Medical Systems) to assess tissue elasticity. Images were generated by slight prostate compression and decompression with the transrectal probe. Hard areas were color-coded blue and considered suspicious for prostate cancer (Fig. 1). These areas had to be reproducible in the axial and sagittal planes according to a previously described approach, and the diameters in all three orientations were measured [15]. The operator was blinded to multiparametric MRI, clinical, and histopathologic findings.

\section{Multiparametric MRI}

Multiparametric MR images were interpreted by an experienced reader. MRI was performed with a 1.5-T system (Avanto, Siemens Healthcare) and a six-channel phased-array body coil combined with an endorectal coil. After digital rectal examination the balloon of the endorectal coil was inflated with $50 \mathrm{~mL}$ of air. A volume calculation for cancer-suspibo spin-echo with variable flip angle (SPACE, Siemens Healthcare) images with the volumetric tool Syngovia (Siemens Healthcare). Each MRI techcious lesions was performed on T2-weighted 3D tur- nique was scored according to how it represented a suspicious prostate cancer lesion. The summary of these points (3-9 points) was the final score. Lesions with more than 5 points were considered to be suspicious for cancer. The reader was blinded to RTE, clinical, and histopathologic findings.

\section{T2-Weighted MRI}

T2-weighted MR images were obtained in the axial and sagittal planes with turbo spin-echo sequences. The entire prostate and the seminal vesicles were investigated. The axial plane was defined by an orientation $90^{\circ}$ to the urethra to be comparable to the histopathologic slices. A SPACE 3D sequence was also performed in the coronal plane. The T2-weighted MRI parameters are shown in Table 1. Low-signal-intensity nodules or ill-defined low-signal-intensity areas in the normally high-signal-intensity peripheral zone or low-signal-intensity areas with ill-defined margins in the transitional zone were considered suspicious for prostate cancer [16]. On T2-weighted MR images the reader assigned a score to the findings using a 3-point scale: 1, benign; 2, intermediate; 3 , malignant. All volumetric measurements were performed on T2-weighted images, which provide the best anatomic information.

\section{TABLE I: Parameters for Multiparametric MRI}

\begin{tabular}{l|c|c|c}
\hline \multicolumn{1}{c|}{ Parameter } & T2 Weighted & Diffusion Weighted & Dynamic Contrast Enhanced \\
\hline Sequence & Fast spin-echo & Spin-echo echoplanar imaging & T1-weighted 3D FLASH \\
TR & 3520 & 2200 & 2.83 \\
TE & 87 & 74 & 1.02 \\
Flip angle $\left(^{\circ}\right)$ & 90 & 90 & 9 \\
FOV $\left(\mathrm{mm}^{2}\right)$ & $180 \times 180$ & $210 \times 210$ & $380 \times 285$ \\
Matrix & $25 \times 256$ & $128 \times 90$ & $256 \times 128$ \\
Slice thickness $(\mathrm{mm})$ & 3 & 3 & 4 \\
b value $\left(\mathrm{s} / \mathrm{mm}^{2}\right)$ & & $50 / 400 / 800$ & $9: 48(60 \times)$ \\
Acquisition time $(\mathrm{min}: \mathrm{s})$ & $1: 54$ & $0: 46$ &
\end{tabular}




\section{Elastography and MRI for Prostate Cancer Detection}

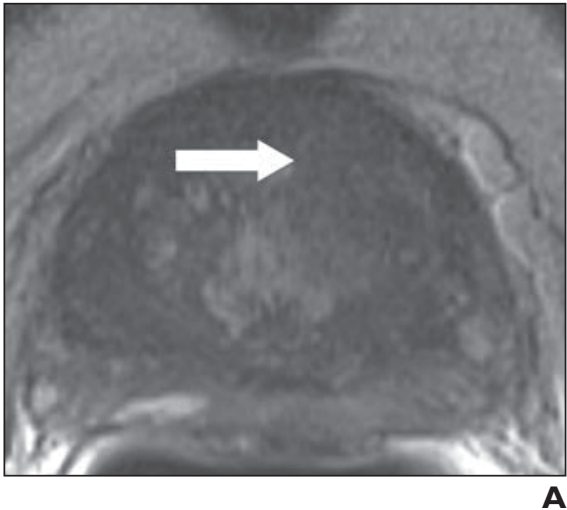

Fig. 2-58-year-old man with prostate cancer (arrow) in left anterior transitional zone.

A-D, Multiparametric T2-weighted low-signalintensity $(\mathbf{A})$ and low apparent diffusion coefficient (B) images and washout curve (C) in hyperperfused area (D) show lesion. $\mathrm{ROI}=$ region of interest.

E, Histologic whole-mount step section shows cancer.

\section{Diffusion-Weighted MRI}

DW images were obtained in axial planes with echoplanar imaging sequences at three $b$ values $\left(50,400\right.$, and $\left.800 \mathrm{~s} / \mathrm{mm}^{2}\right)$. Restriction of diffusion was quantified by apparent diffusion coefficient (ADC) value. The DWI parameters are shown in Table 1. On DW images the reader assigned a score to imaging findings using a 3-point scale according to ADC value: 1, benign at ADC > 1200; 2 , intermediate at ADC 900-1200; 3, malignant at $\mathrm{ADC}<900$ [16].

\section{Contrast-Enhanced MRI}

Contrast-enhanced MR images were obtained in axial planes with fast 3D T1-weighted volumetric interpolated breath-hold examination (VIBE) gradient-echo sequences every 7 seconds for approximately 10 minutes during continuous injection of gadolinium contrast material (gadoterate meglumine, Dotarem, Guerbet; flow rate, 0.1 $\mathrm{mL} / \mathrm{s})$. The CE-MRI parameters are shown in Table 1. Perfusion curves were generated with the Tissue4D program (Siemens Healthcare), which calculates a pharmacokinetic model derived from the Tofts model. Pharmacokinetic variables, including $\mathrm{K}^{\text {trans }}$ and $\mathrm{K}_{\mathrm{ep}}$, were determined [16]. Ar-
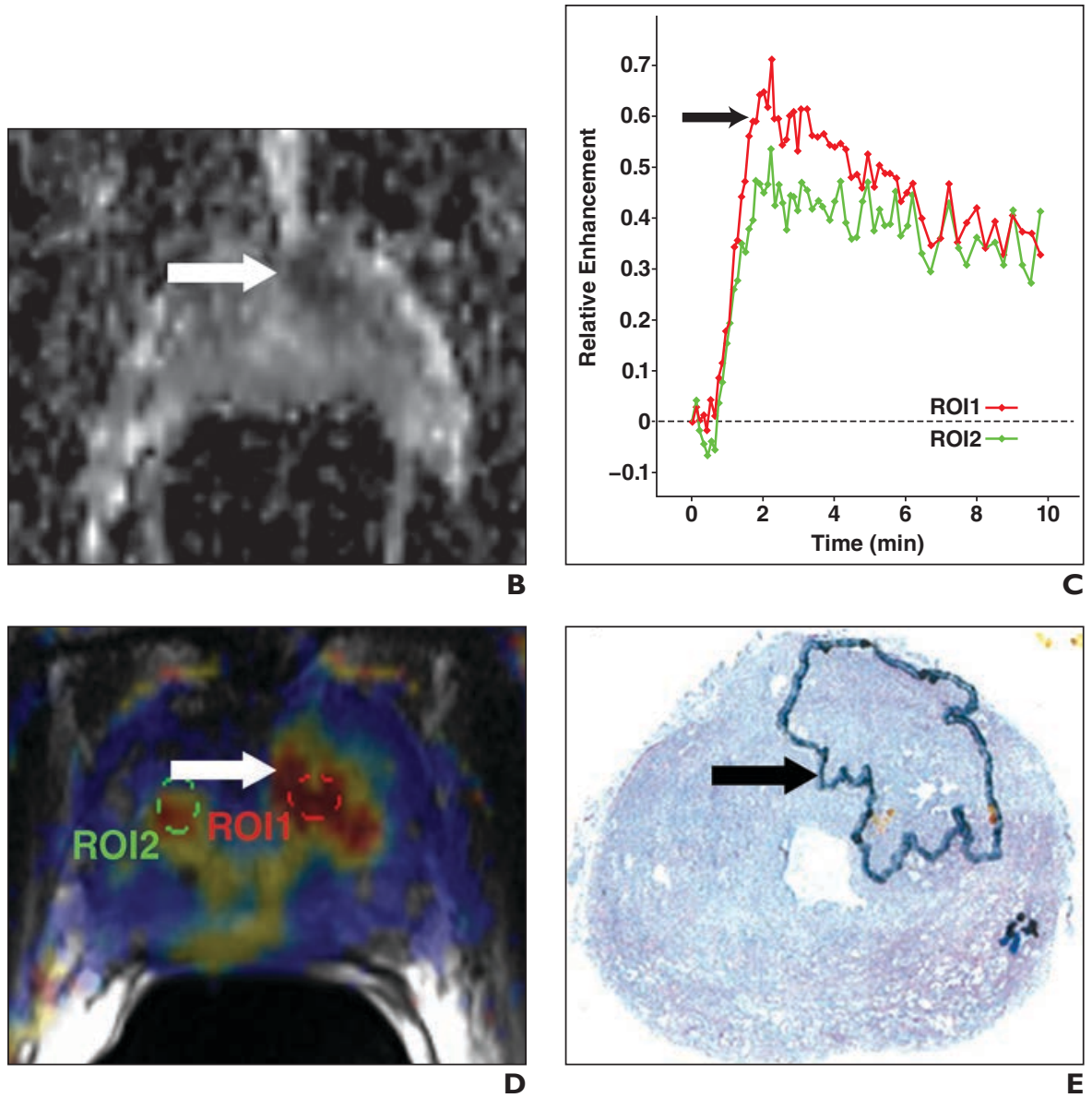

eas with increased time to peak, increased peak enhancement, and increased washout rate were considered suspicious for prostate cancer. Asymmetric hyperperfusion or hyperperfusion within a focal lesion was considered highly suspicious. On CE-MR images, the reader assigned a score to imaging findings using a 3 -point scale: 1 , benign; 2 , intermediate; 3, malignant (Fig. 2).

\section{Histopathologic Preparation, Reporting, and Correlation With Imaging Findings}

After radical prostatectomy and fixation, the prostatectomy specimens were laminated in 4-mm-thick slices with an orientation of $90^{\circ}$ to the urethra. Pathologic analysis was performed by an experienced pathologist, who marked all cancer lesions and reported the Gleason score assigned. A shrinkage factor of $10 \%$ was into account for assessment of tumor volumes. The prostate of each patient was divided into peripheral and transitional zone and into anterior, posterior, right, and left parts. The border between the anterior and posterior parts was defined as the imaginary line through the widest transverse diameter of the prostate. Only cancer lesions with a volume of $0.2 \mathrm{~cm}^{3}$ or more were considered for analysis.

\section{Statistical Analysis and Measurements}

For all patients, summary statistics were provided with the appropriate measures of location and measures of variation. Only areas suspicious for prostate cancer with a volume of $0.2 \mathrm{~cm}^{3}$ or greater were considered for statistical analysis. Detection rates including exact $95 \%$ CIs were calculated for multiparametric MRI and RTE. The Fisher exact test was used for comparison. The measurements of tumor volumes were compared between histopathologic slices, RTE, and T2weighted MRI by Spearman rank correlation and exact $95 \%$ CIs. All statistical calculations were performed with SPSS software (version 18.0, IBM SPSS), and $p<0.05$ was considered statistically $p>0.03$ (anatomic regions of the prostate, prostate volume, tumor volume, and Gleason score) were interpreted as very conservative. Multivariate analysis was performed; all combinations considered relevant are listed in Table 2.

\section{Results}

Histologic examination of the 39 patients who underwent prostatectomy revealed 61 cancer lesions with a volume of $0.2 \mathrm{~cm}^{3}$ or greatsignificant. All findings of subgroup analyses with 
Junker et al.

TABLE 2: Prostate Cancer Detection Rates Based on Localization, Tumor Volume, Gleason Score, and Prostate Volume

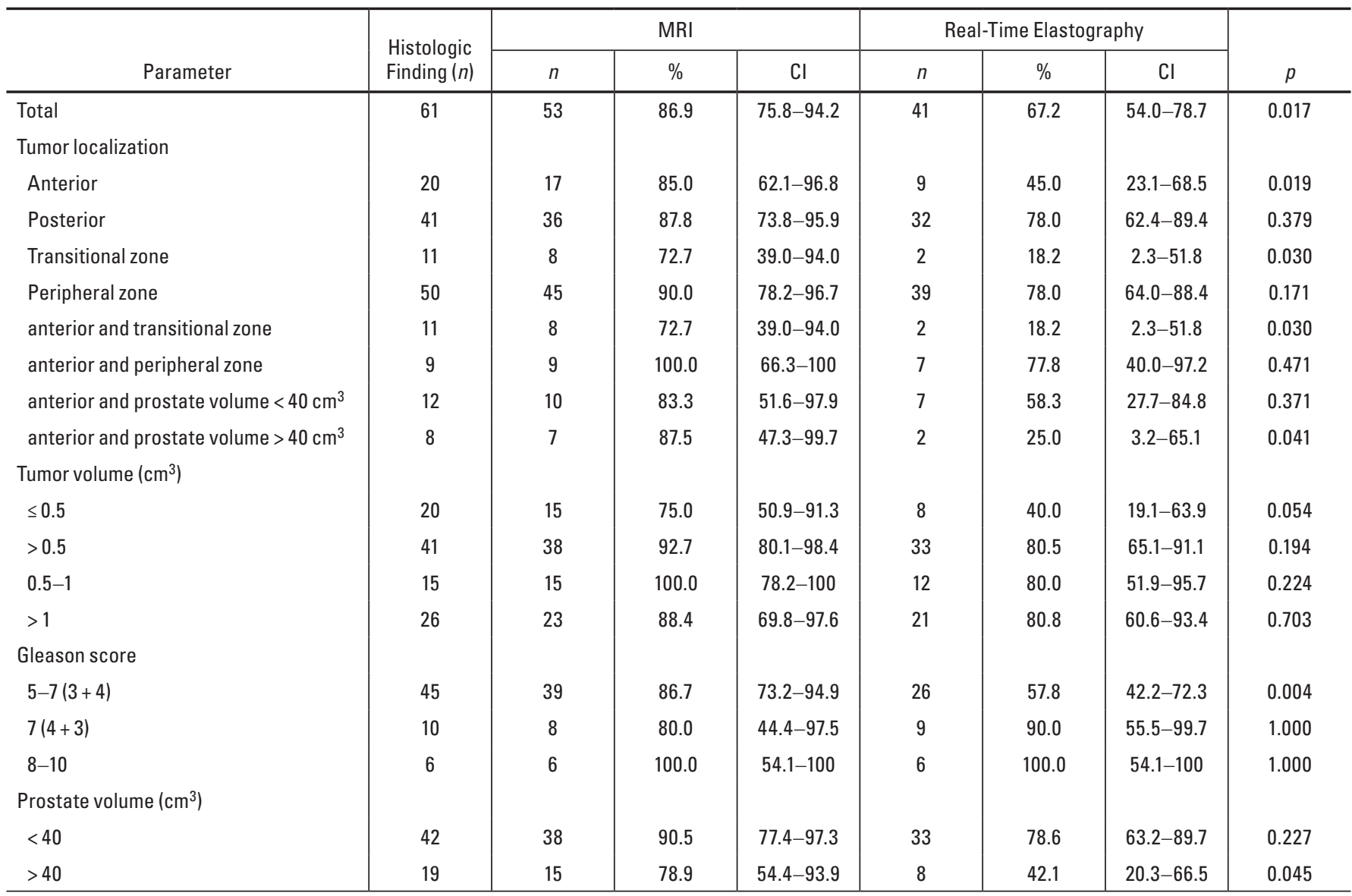

er (median, $0.85 \mathrm{~cm}^{3}$; range, $0.2-11.18 \mathrm{~cm}^{3}$ ), of which $50(82.0 \%)$ were localized in the peripheral zone, $11(18.0 \%)$ in the transitional zone, $20(32.9 \%)$ in the anterior part, and $41(67.2 \%)$ in the posterior part of the prostate. The median Gleason score was 7 (range, 5-10).

\section{Prostate Cancer Detection Rates According to Localization and Prostate Volume}

In total, RTE depicted 41 of 61 cancer lesions $(67.2 \%)$ with a false-positive rate of $25.5 \%$. Multiparametric MRI depicted 53 of 61 lesions $(86.9 \%)$ with a false-positive rate of $13.1 \%$ ( $p=0.017$ ) (Table 2). The combined approach of RTE and MRI depicted 56 of 61 cancer lesions $(91.8 \%)$.

When cancer lesions were located in the posterior parts of the prostate, there was no significant difference in prostate cancer detection rate between RTE $(78.0 \%)$ and multiparametric MRI (87.8\%) ( $p=0.379)$. A significant difference in prostate cancer detection rate was found in the anterior parts with an overall sensitivity of $45.0 \%$ for RTE and $85.0 \%$ for multiparametric MRI ( $p=$
0.019). This difference was mainly caused by coexistent transitional zone localization or prostate volumes greater than $40 \mathrm{~cm}^{3}$. However, no significant difference between the two imaging modalities was found when anterior cancers were located in the peripheral zone $(p=0.471)$ or in prostates with volumes less than $40 \mathrm{~cm}^{3}(p=0.371)$ (Table 2). Regarding the peripheral zone only, the pros- tate cancer detection rates for RTE (78.0\%) and multiparametric MRI $(90.0 \%)$ did not differ significantly $(p=0.171)$.

\section{Prostate Cancer Detection Rates According to} Tumor Volumes

Irrespective of tumor volumes, the two modalities had similar detection rates. No significant differences were found. Within each mo-

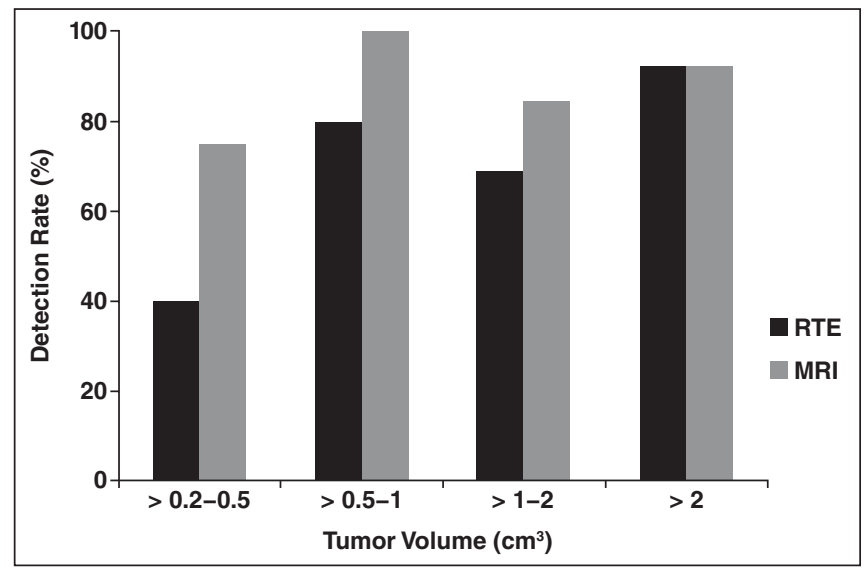

Fig. 3-Graph shows prostate cancer detection rate according to tumor volume. $\mathrm{RTE}=$ real-time elastography. 


\section{Elastography and MRI for Prostate Cancer Detection}

dality the detection rates for tumor volumes greater than $0.5 \mathrm{~cm}^{3}$ compared with volumes of $0.5 \mathrm{~cm}^{3}$ or less differed significantly for RTE $(p<0.003)$ but not for multiparametric MRI $(p=0.100)$ (Fig. 3 and Table 2).

\section{Prostate Cancer Detection According to Gleason Score}

For high-risk prostate cancer (Gleason score $\geq 4+3)$, RTE had a detection rate of $93.8 \%$ and MRI of $87.5 \%(p=1.0)$. A significant difference between modalities was found for the detection of low-risk prostate cancer (Gleason score $\leq 3+4$ ) with detection rates of $57.8 \%$ for RTE and $86.7 \%$ for MRI ( $p=$ $0.004)$. In contrast to that of multiparametric MRI, the detection rate of RTE within the modality was significantly higher for cancer lesions with a predominant Gleason pattern $\geq$ 4 than for a pattern $\leq 3$ ( $p=1.000$ for multiparametric MRI, $p<0.012$ for RTE).

\section{Comparison of Volumetric Measurements}

Overall the median cancer volume was $0.82 \mathrm{~cm}^{3}$ (range, $0.21-11.2 \mathrm{~cm}^{3}$ ). Spearman rank correlation with histopathologic results showed a correlation coefficient of 0.72 (CI, 0.57-0.82) for MRI and 0.46 (CI, 0.24-0.64)

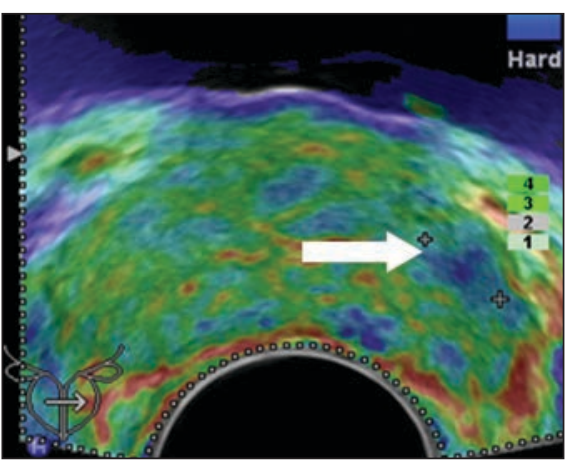

A

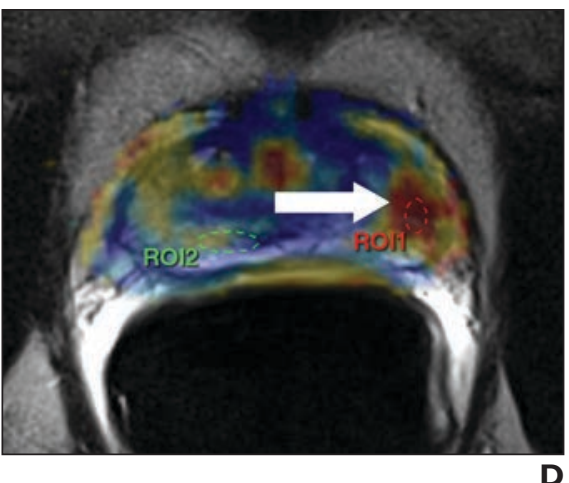

for RTE. Because the CIs do not cover the particular correlation coefficient, this difference has to be regarded as significant.

\section{Discussion}

In general, imaging of prostate cancer is limited by tumor volume (size) and grade, because cancers with a predominant Gleason pattern of 3 (i.e., Gleason score of 6 with $3+$ 3 or of 7 with $3+4$ ) are intermixed with normal glands and glands with dilated lumina (sparse tumors) [7, 17, 18]. Delongchamps et al. [16] used a similar multiparametric MRI setting with an endorectal coil at $1.5 \mathrm{~T}$ and for prostate cancers with volumes greater than $0.2 \mathrm{~cm}^{3}$ reported a sensitivity of $80 \%$ for the peripheral zone and $53 \%$ for the transitional zone, which are in concordance with our results. Furthermore, Yerram et al. [19] found that the presence of only low-suspicion lesions at multiparametric MRI can almost exclude the presence of high-risk prostate cancer. Delongchamps et al. [20] did not miss a single case of high-risk prostate cancer in a study of targeted MRI-ultrasound fusion biopsy. These data suggest that clinically significant disease can be detected with high confidence with imaging techniques
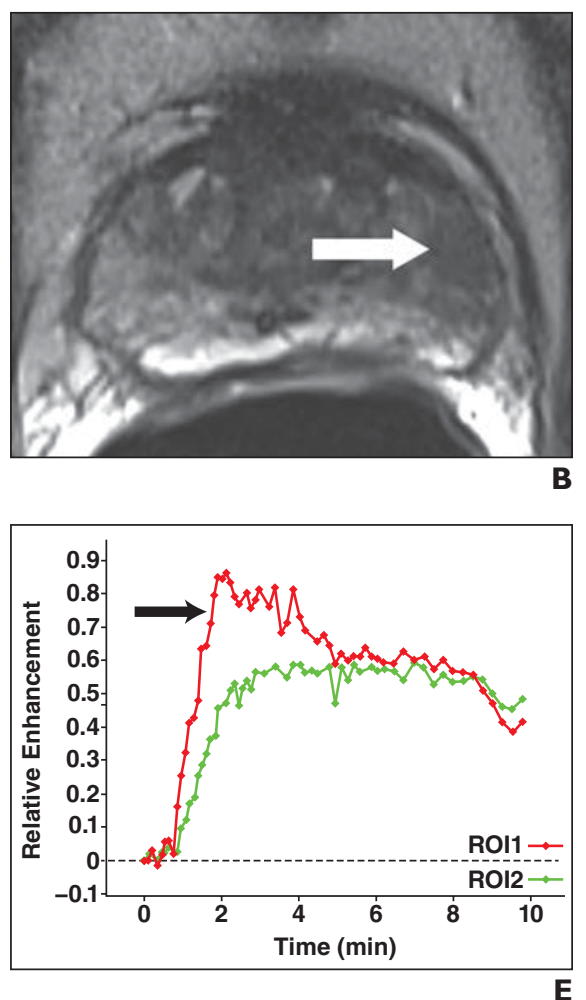

and that therefore these techniques can be reliable tools for clinicians dealing with prostate cancer diagnosis and therapy. Therefore, the most important issue of this study was to compare RTE and multiparametric MRI regarding their accuracy in detection and characterization of clinically significant disease.

Tumor volume is one important factor for defining significant disease. We found comparably high detection rates for prostate cancers with volumes greater than $0.5 \mathrm{~cm}^{3}$ for both RTE $(80.5 \%)$ and multiparametric MRI $(92.7 \%)$ (Fig. 4). In accordance with the studies by Sumura et al. [14] and Roethke et al. [18] dealing with detection rates in relation to tumor volume, in our study both modalities had increasing accuracy for prostate cancer detection with increasing tumor volume. Sumura et al., to our knowledge the first study group to evaluate RTE and MRI in the same patient population, found superiority for RTE in the detection of small cancer lesions compared with T2-weighted MRI and CE-MRI separately. In comparison with their findings, our results suggest that combining T2-weighted MRI, CE-MRI, and DWI increases sensitivity for the detection of small cancer lesions in multiparametric MRI. This may explain
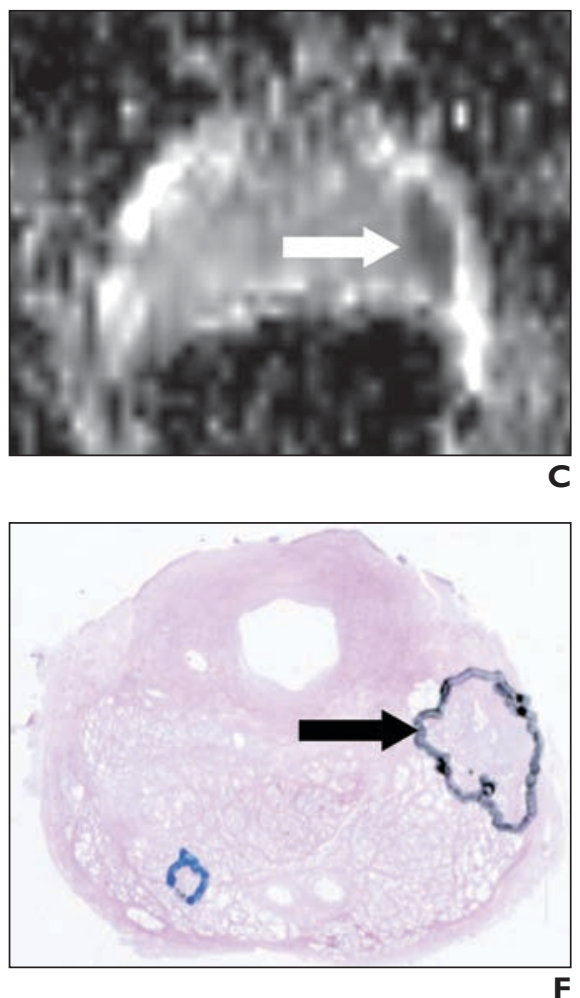

Fig. 4-65-year-old man with prostate cancer.

A-F, Axial real-time elastogram (A), T2 -weighted low-signal intensity (B), and low apparent diffusion coefficient (C) MR images, washout curve (E) in hyperperfused area (D), and histologic whole-mount step section (F) show clinically significant prostate cancer (arrow) midgland in left peripheral zone. ROI = region of interest. 
our observation that the diagnostic accuracy of RTE was more dependent on tumor size than was the accuracy of MRI. This agrees with our observation that Spearman rank correlation was 0.72 for multiparametric MRI and only 0.46 for RTE in comparisons of volume measurements of cancer lesions with the histopathologic findings.

According to our findings, multiparametric MRI seems more reliable for tumor volume assessment. Thus for active surveillance of suspicious lesions and for follow-up examinations to detect tumor growth, MRI seems to be more reliable. This is in line with the findings of Curiel et al. [21], who investigated an ultrasonic elastographic imaging system that may provide a simple and cost-effective solution to monitoring high-intensity focused ultrasound treatments [21]. They assessed the volume of high-intensity focused ultrasound lesions in prostates and found that in general tumor volume was underestimated with elastography in comparison with MRI.

Regarding tumor histology, in the current study comparably high rates of detection of high-risk prostate cancer were found for RTE with (93.8\%) and multiparametric MRI $(87.5 \%)$. The one case of high-risk prostate cancer missed with RTE was located in the transitional zone, and the two cases missed with multiparametric MRI were not visible because of artifacts of the endorectal coil. Pelzer et al. [12], who also compared RTE findings with multiparametric MRI findings for prostate cancer diagnosis, found a reduced rate of detection of low-risk prostate cancer for both methods compared with detection of high-risk prostate cancer. Slightly different from these findings, in our study population with low serum prostate-specific serum concentrations, multiparametric MRI was superior to RTE for visualization of low-risk prostate cancer $(86.7 \%$ for multiparametric MRI and $57.8 \%$ for RTE; $p<0.005)$. This finding might have occurred because RTE alone can provide information about tissue hardness only. In contrast, multiparametric MRI reveals structural and tissue perfusion information. The usefulness of combining RTE with contrast-enhanced ultrasound has been found in previous studies [6, 22].

Like those of Pelzer et al. [12], our results suggest that multiparametric MRI may have advantages in the detection of transitional zone cancers. The sensitivities were $72.7 \%$ for multiparametric MRI and $18.2 \%$ for RTE $(p<$ 0.05 ). MRI also seems superior for visualization of anterior cancers located in prostates with a volume greater than $40 \mathrm{~cm}^{3}$. These results may be explained by cancer localization and the technical limits of RTE: All transitional zone cancers were highly anterior, and when calcifications were simultaneously located in the posterior parts of the transitional zone, there was no possibility of reaching these regions for RTE and ultrasound. In general this is due to hardening artifacts. In addition, investigation of the transitional zone and anterior parts in prostates with benign prostatic hypertrophy is limited for RTE because of stiffness artifacts of the inner gland [23]. However, RTE showed no significant difference in the detection of these tumors compared with multiparametric MRI when anterior cancers were located in prostates with a volume less than $40 \mathrm{~cm}^{3}$ $(p=0.37)$ or in the peripheral zone only ( $p=$ $0.45)$. This observation was also made for the dorsal parts of the peripheral zone $(p=0.38)$ in comparisons of the two modalities. Moreover, with one exception all transitional zone cancers were low-risk cancers, and significant differences in prostate cancer detection between RTE and multiparametric MRI in these regions existed only for cancer lesions $0.5 \mathrm{~cm}^{3}$ and smaller. These findings suggest that investigation of the inner gland and of anterior regions of the prostate, although less reliable than MRI, can be accurately performed with RTE in prostates with a volume less than 40 $\mathrm{cm}^{3}$ and for detection of high-risk tumors by a skilled investigator.

Our study had several limitations. We did not have data about intraobserver and interobserver variability for the two methods. Second, we focused this study on correlation with histopathologic whole-mount step sections, and because only patients with confirmed cancer undergo radical prostatectomy it was not possible to perform a correlation with negative results. Third, for the same reason, we knew that every patient had prostate cancer, which is a bias. Fourth, there was no direct image-to-image registration for the original datasets. Therefore, we are not ultimately sure whether the identical geographic areas were compared for volumetric analysis. Fifth, in some patients the endorectal coil used for multiparametric MRI caused extinction artifacts in posterior regions of the prostate. Thus two cancer lesions were missed with multiparametric MRI that may have been detected without the endorectal coil. Sixth, MRI was performed in a multiparametric way, whereas ultrasound was performed with RTE alone. A prospective comparison between multiparametric MRI and multiparametric ultrasound (i.e., B-mode, contrast-enhanced ultrasound, and RTE) would be interesting. The final three limitations were the modest sample size, performance of a single evaluation of all individuals (reproducibility is unknown) and single pathologist observer, and the variable time between biopsy and imaging.

Summarizing our results and considering that in contrast to multiparametric MRI, RTE is a cost-effective and noninvasive technique that can be performed under real-time conditions, the integration of the two methods in clinical routine can be achieved as proposed by Heijmink et al. [24]: Because RTE has similar good reliability for the diagnosis of high-risk cancer, it seems sufficient for screening purposes and for initial biopsies, in which RTE-targeted cores can be obtained in addition to systematic cores. Several studies have shown that this combined approach may raise the overall sensitivity in prostate cancer diagnosis $[9,25]$. If biopsy results are negative but there is ongoing suspicion of prostate cancer, the limitations of RTE, especially for the detection of transitional zone tumors and anteriorly located tumors in prostates with benign prostatic hypertrophy, should be remembered. These patients then may undergo multiparametric MRI to achieve the high sensitivity of the combined approach as found in this study. If lesions suspicious for being prostate cancer are found at multiparametric MRI, they can be biopsied with multiparametric MRI targeting or with cognitive or technical fusion by means of ultrasound [26-28].

\section{References}

1. Heidenreich A, Bastian PJ, Bellmunt J, et al. Guidelines on prostate cancer. Arnheim, The Netherlands: European Association of Urology, 2012:17

2. Djavan B, Ravery V, Zlotta A, et al. Prospective evaluation of prostate cancer detected on biopsies 1, 2, 3 and 4: when should we stop? J Urol 2001; 166:1679-1683

3. Schlemmer HP. Multiparametric MRI of the prostate: method for early detection of prostate cancer? Rofo 2010; 182:1067-1075

4. Durmus T, Stephan C, Grigoryev M, et al. Detection of prostate cancer by real-time MR/ultrasound fusion-guided biopsy: 3T MRI and state of the art sonography [in German]. Rofo 2013; 185:428-433

5. Panebianco V, Sciarra A, Marcantonio A, et al. Conventional imaging and multiparametric magnetic resonance (MRI, MRS, DWI, MRP) in the diagnosis of prostate cancer. $Q \mathrm{~J} \mathrm{Nucl} \mathrm{Med} \mathrm{Mol}$ Imaging 2012; 56:331-342 


\section{Elastography and MRI for Prostate Cancer Detection}

6. Aigner F, Schäfer G, Steiner E, et al. Value of enhanced transrectal ultrasound targeted biopsy for prostate cancer diagnosis: a retrospective data analysis. World J Urol 2012; 30:341-346

7. Junker D, Schäfer G, Aigner F, et al. Potentials and limitations of real-time elastography for prostate cancer detection: a whole-mount step section analysis. ScientificWorldJournal 2012; 1012:193213

8. Krouskop TA, Wheeler TM, Kallel F, Garra BS, Hall T. Elastic moduli of breast and prostate tissues under compression. Ultrason Imaging 1998; 20:260-274

9. Pallwein L, Mitterberger M, Pinggera G, et al. Sonoelastography of the prostate: comparison with systematic biopsy findings in 492 patients. Eur $J$ Radiol 2008; 65:304-310

10. Kurhanewicz J, Vigneron D, Carroll P, Coakley F. Multiparametric magnetic resonance imaging in prostate cancer: present and future. Curr Opin Urol 2008; 18:71-77

11. Barentsz JO, Richenberg J, Clements R, et al. ESUR prostate MR guidelines 2012. Eur Radiol 2012; 22:746-757

12. Pelzer AE, Heinzelbecker J, Weiß C, et al. Realtime sonoelastography compared to magnetic resonance imaging using four different modalities at 3.0 $\mathrm{T}$ in the detection of prostate cancer: strength and weaknesses. Eur J Radiol 2013; 82:814-821

13. Aigner F, Pallwein L, Schocke M, et al. Comparison of real-time sonoelastography with T2weighted endorectal magnetic resonance imaging for prostate cancer detection. J Ultrasound Med 2011; 30:643-649

14. Sumura M, Shigeno K, Hyuga T, Yoneda T, Shiina
H, Igawa M. Initial evaluation of prostate cancer with real-time elastography based on step-section pathologic analysis after radical prostatectomy: a preliminary study. Int J Urol 2007; 14:811-816

15. König K, Scheipers U, Pesavento A, Lorenz A, Ermert H, Senge T. Initial experiences with realtime elastography guided biopsies of the prostate. J Urol 2005; 174:115-117

16. Delongchamps NB, Rouanne M, Flam T, et al Multiparametric magnetic resonance imaging for the detection and localization of prostate cancer: combination of T2-weighted, dynamic contrastenhanced and diffusion-weighted imaging. $B J U$ Int 2011; 107:1411-1418

17. Langer DL, Van Der Kwast TH, Evans AJ, et al. Intermixed normal tissue within prostate cancer: effect on MR imaging measurements of apparent diffusion coefficient and T2-sparse versus dense cancers. Radiology 2008; 249:900-908

18. Roethke MC, Lichy MP, Jurgschat L, et al. Tumor size dependent detection rate of endorectal MRI of prostate cancer: a histopathologic correlation with whole-mount sections in 70 patients with prostate cancer. Eur J Radiol 2011; 79:189-195

19. Yerram NK, Volkin D, Turkbey B, et al. Low suspicion lesions on multiparametric magnetic resonance imaging predict for the absence of high-risk prostate cancer. BJU Int 2012; 110(11 pt B):E783-E788

20. Delongchamps NB, Peyromaure M, Schull A, et al. Prebiopsy magnetic resonance imaging and prostate cancer detection: comparison of random and targeted biopsies. J Urol 2013; 189:493-499

21. Curiel L, Souchon R, Rouvière O, Gelet A, Chapelon JY. Elastography for the follow-up of high-intensity focused ultrasound prostate cancer treatment: initial comparison with MRI. Ultrasound Med Biol 2005; 31:1461-1468

22. Brock M, Eggert T, Palisaar RJ, et al. Multiparametric ultrasound of the prostate: adding contrast enhanced ultrasound to real-time elastography to detect histopathologically confirmed cancer. $J$ Urol 2013; 189:93-98

23. Pallwein L, Aigner F, Faschingbauer R, et al. Prostate cancer diagnosis: value of real-time elastography. Abdom Imaging 2008; 33:729-735

24. Heijmink SW, Fütterer JJ, Strum SS, et al. Stateof-the-art uroradiologic imaging in the diagnosis of prostate cancer. Acta Oncol 2011; 50(suppl 1):25-38

25. Brock M, von Bodman C, Palisaar RJ, et al. The impact of real-time elastography guiding a systematic prostate biopsy to improve cancer detection rate: a prospective study of 353 patients. $J$ Urol 2012; 187:2039-2043

26. Smeenge M, Barentsz J, Cosgrove D, et al. Role of transrectal ultrasonography (TRUS) in focal therapy of prostate cancer: report from a consensus panel. BJU Int 2012; 110:942-948

27. Park BK, Park JW, Park SY, et al. Prospective evaluation of 3-T MRI performed before initial transrectal ultrasound-guided prostate biopsy in patients with high prostate-specific antigen and no previous biopsy. AJR 2011; 197:[web]W876W881

28. Schilling D, Kurosch M, Mager R, Tsaur I, Haferkamp A, Röthke M. Fusion imaging in urology: combination of MRI and TRUS for detection of prostate cancer. Urologe A 2013; 14:481-489 